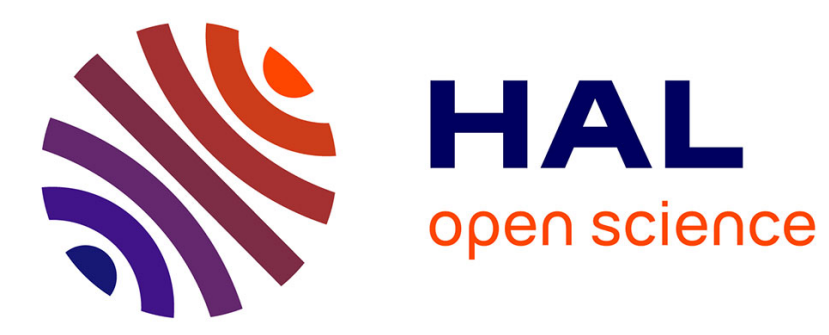

\title{
Évolution de l'état sanitaire et stratégies de subsistance des populations protohistoriques en Arabie orientale Olivia Munoz
}

\section{To cite this version:}

Olivia Munoz. Évolution de l'état sanitaire et stratégies de subsistance des populations protohistoriques en Arabie orientale. Froment, Alain; Guy, Hervé. Archéologie de la santé, anthropologie du soin, Editions La Découverte; Inrap, pp.95-106, 2019, Recherches, 978-2-348-04577-6. hal-02359181

\section{HAL Id: hal-02359181 \\ https://hal.science/hal-02359181}

Submitted on 12 Nov 2019

HAL is a multi-disciplinary open access archive for the deposit and dissemination of scientific research documents, whether they are published or not. The documents may come from teaching and research institutions in France or abroad, or from public or private research centers.
L'archive ouverte pluridisciplinaire HAL, est destinée au dépôt et à la diffusion de documents scientifiques de niveau recherche, publiés ou non, émanant des établissements d'enseignement et de recherche français ou étrangers, des laboratoires publics ou privés. 


\title{
Évolution de l'état sanitaire et stratégies de subsistance des populations protohistoriques en Arabie orientale
}

\begin{abstract}
Olivia Munoz ${ }^{1}$
Dans l'imaginaire collectif, l'Arabie est perçue comme un vaste territoire désertique, parsemé de quelques oasis où des bédouins nomades font halte pour abreuver leurs dromadaires. Pourtant, les premiers systèmes agricoles oasiens n'ont fait leur apparition dans cette région qu'aux environs de 3000 avant notre ère [TENGBERG, 2012] et les dromadaires ne semblent avoir été domestiqués pour le transport qu'au tournant du Ir millénaire avant notre ère [UERPMANN et UERPMANN, 2002].

Les recherches archéologiques menées depuis une quarantaine d'années en Arabie orientale ont mis en évidence les transformations socio-économiques importantes qui caractérisent la transition entre le Néolithique (6500-3100 avant notre ère environ) et l'âge du Bronze ancien (3100-2000 avant notre ère environ) dans un contexte continu d'aridification. Face à de fortes contraintes environnementales, et en comparaison des régions alentours, comme la Mésopotamie, l'Iran ou la vallée de l'Indus, ces sociétés ont suivi des trajectoires évolutives originales. L'élevage y est pratiqué autour de 7000-6500 avant notre ère par des communautés acéramiques et assez mobiles. Les premières évidences d'activités agricoles n'apparaissent que quatre mille ans plus tard, au début de l'âge du Bronze [Cleuziou, 2005 ; Cleuziou et Tosi, 2007 ; CHARBONNIER, 2017].

Cet article propose, après une brève présentation du contexte géographique et environnemental de la zone d'étude, une synthèse des conditions de vie des groupes humains de cette région aride, au Néolithique puis à l'âge du Bronze ancien, et précise l'état sanitaire de ces populations, à partir des données issues de plusieurs sites emblématiques. Néanmoins, du fait de conditions de conservation peu favorables aux restes osseux, il est important de souligner que la qualité de la documentation ostéologique à disposition des bioanthropologues est, dans son ensemble, lacunaire et d'assez mauvaise qualité.
\end{abstract}

\section{Un environnement contraignant et des ressources morcelées}

L'Arabie orientale est géographiquement très diversifiée : bordée par le golfe Persique au nord-ouest, le golfe d'Oman au nord-est et la mer d'Arabie à l'est, elle est traversée sur environ sept cents kilomètres par un important système montagneux, la chaîne du Hajjar, qui forme une véritable barrière entre la mer et l'intérieur des terres avec très peu de passages naturels. À l'ouest, la péninsule s'ouvre sur l'immense désert du Rub'al-Khâli (fig. 1). Le territoire est ainsi morcelé en une variété de niches écologiques exploitables, des environnements côtiers (qui occupent $3 \%$ du territoire), aux zones montagneuses (15\%), en passant par les piémonts et les déserts (82\%).

${ }^{1}$ CNRS, UMR 7041, Maison de l'Archéologie et de l'Ethnologie de Nanterre, France 
Actuellement, le climat de l'Arabie orientale est caractérisé par une très forte aridité, avec des précipitations moyennes inférieures à $100 \mathrm{~mm}$, des températures très élevées et une évaporation considérable. Pendant l'Holocène, le climat a connu des fluctuations importantes, et globalement, après une phase humide amorcée il y a environ dix mille ans, il s'est aridifié progressivement autour de 4000 avant notre ère [LÉZINE et al., 2010].

Du fait de ces conditions arides, les ressources terrestres sont morcelées, assez limitées et disponibles de façon saisonnière, en fonction des précipitations. Pour faire face au morcellement des ressources, les populations traditionnelles ont fondé leur économie sur trois composantes correspondant aux différentes niches écologiques : l'élevage, dans les zones steppiques, l'agriculture dans les zones où l'eau affleure, dans les vallées et les piémonts, et la pêche sur les côtes. Ce modèle traditionnel repose à la fois sur une mobilité saisonnière des groupes et sur la mise en place de systèmes d'échanges entre zones complémentaires. Mais qu'en a-t'il été pendant la protohistoire?

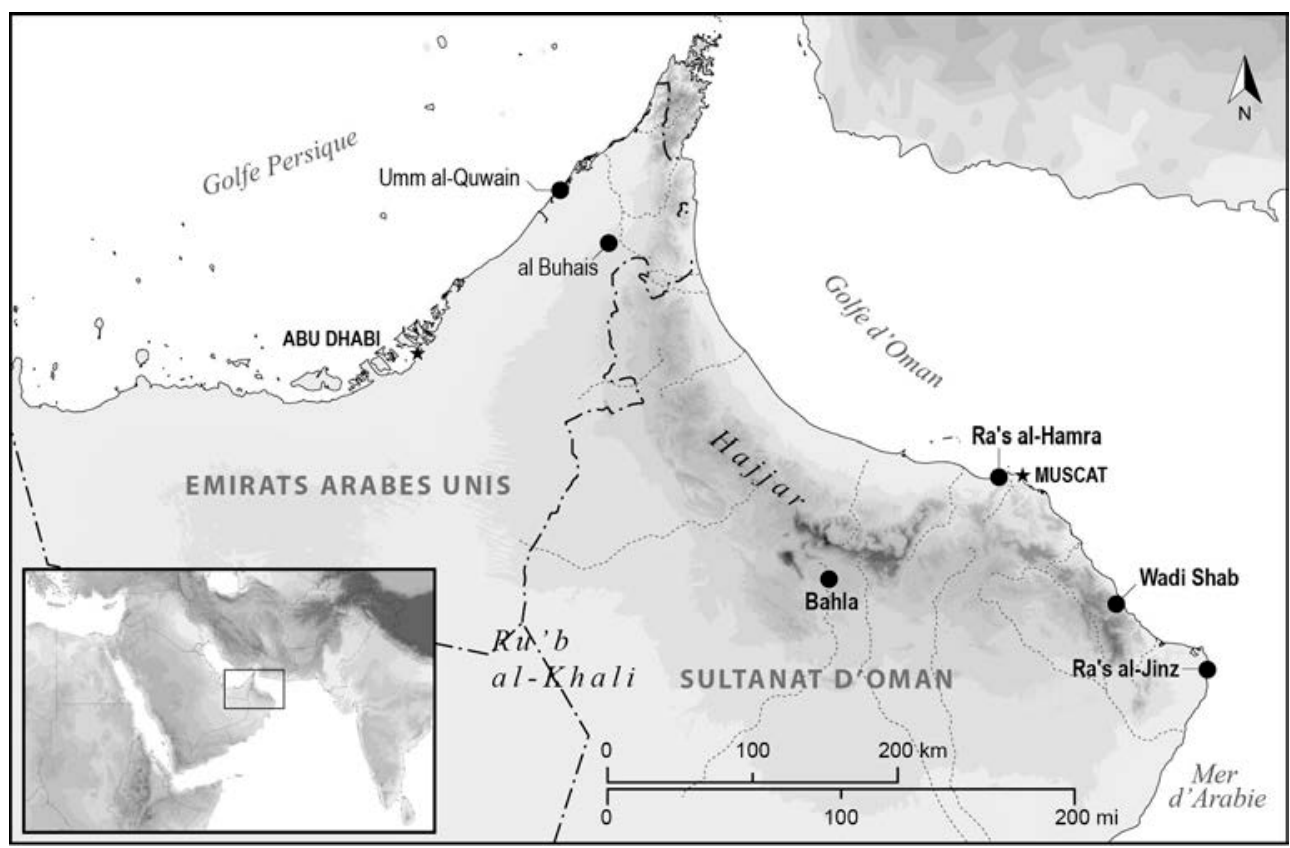

Figure 1 : Localisation des sites mentionnés dans la péninsule Arabique (Carte O. Munoz).

\section{Les communautés néolithiques : des pasteurs...}

Les données disponibles à l'heure actuelle indiquent qu'avant que la maîtrise de techniques d'irrigation sophistiquées autorise le développement de l'agriculture d'oasis, l'économie des groupes occupant l'intérieur des terres était principalement basée sur un pastoralisme nomade - l'élevage de bovins, de moutons et de chèvres étant attesté dès le VI millénaire avant notre ère. Les pointes de flèches en silex retrouvées près d'anciens points d'eau et les assemblages de faunes de sites de plaines et de piémonts révèlent que la chasse aux animaux sauvages pouvait également contribuer à la subsistance de ces communautés [BEECH et al., 2009 ; UERPMANN et al., 2008]. L'occupation humaine semble avoir été plus intense sur le littoral, où les ressources côtières et des niches écologiques favorables (mangroves et lagunes) constituent des pôles très attractifs.

Dans les zones de piémonts, on suppose que c'est la présence de sources d'eau et de pâturages ce qui a favorisé 
les installations pendant des épisodes climatiques humides. Ainsi, par exemple, le site majeur connu à l'intérieur des terres, al-Buhais 18, situé dans l'émirat de Sharjah, comporte une source d'eau aujourd'hui fossile, qui a probablement attiré les populations anciennes. Les fouilles menées par Hans-Peter Uerpmann et son équipe ont livré les vestiges de fosses, de foyers et des déchets domestiques, installés autour d'un vaste ensemble sépulcral utilisé autour de 4500 avant notre ère, où près de six cents individus ont été inhumés [UERPMANN et al., 2006 ; 2008]. D'après les restes d'animaux retrouvés, la subsistance du groupe était principalement assurée par l'élevage (95\%), principalement des moutons et des chèvres, et en moindre proportion des bovins. La part réduite des animaux sauvages - âne, dromadaire, oryx, gazelle - indique que la chasse jouait un rôle mineur sur ce site. L'étude des vestiges dentaires humains complète les données archéozoologiques : elle révèle une alimentation faible en glucides (lactose, fructose, glucose) et de texture abrasive (on ne relève aucune carie, très peu de pertes ante-mortem de dents $(2 \%)$ ou d'abcès $(1,2 \%)$, et un fort taux d'usure de l'émail dentaire [KIESEWETTER, 2006]).

Des analyses isotopiques menées sur les individus inhumés à al-Buhais indiquent que le site était une station saisonnière au sein d'un circuit de pastoralisme mobile, dont les autres étapes étaient localisées dans les montagnes [KUTTERER et UERPMANN, 2017]. Certains indices permettent de reconstituer ces déplacements saisonniers : la courbe de mortalité des moutons et des chèvres indique que les groupes n'occupaient le site qu'au printemps. De plus, des graviers de wadi, provenant des montagnes du Hajjar, ont été retrouvés dans plusieurs sépultures secondaires, et suggèrent aux fouilleurs que le traitement primaire des cadavres a eu lieu dans d'autres stations, proches des wadis de montagnes [KUTTERER, 2010]. Des liaisons avec la côte sont également perceptibles à travers les nombreux coquillages des parures et des accessoires vestimentaires ornant les morts dans les tombes.

À la fin du $V^{e}$ millénaire, le site est abandonné et l'intérieur des terres semble alors déserté, peut-être en raison de l'aridification du climat qui aurait compromis ce mode de vie basé sur un pastoralisme nomade [UERPMANN, 2003].

\section{...et des pêcheurs}

À cette période, l'occupation semble s'intensifier le long des côtes du golfe d'Oman et de la mer d'Arabie, où les gisements sont principalement constitués d'amas coquilliers. Les populations fréquentaient des zones présentant des écosystèmes variés, comme l'embouchure des wadis, où la présence de mangroves et de lagunes permettait l'acquisition de nombreuses ressources. C'est le cas de la douzaine de sites distribués sur le promontoire de Ra's al-Hamra et dans la mangrove de Qurum, à Mascate (capitale du sultanat d'Oman). Dans cette zone, l'occupation humaine est continue à partir du milieu du VI millénaire et $s^{\prime}$ intensifie à partir du $\mathrm{V}^{\mathrm{e}}$ avant notre ère.

L'un de ces gisements, Ra's al-Hamra 5, daté du IV millénaire avant notre ère [MARCUCCI et al., 2011], a livré les traces de nombreuses structures domestiques (huttes, cabanes, pare-vents, fosses et foyers) ainsi que des aires d'activités artisanales liées à la pêche. Un important ensemble sépulcral a également été mis au jour, avec près de cent cinquante tombes fouillées à ce jour. Il s'agit de sépultures en fosse, où les morts sont déposés sur le côté, dans une position dite «foetale » [SALVATORI, 2007 ; MUNOZ, 2014].

La culture matérielle montre une variété d'outils dédiés à la pêche ou à la capture d'animaux marins : hameçons, poids de filets, pointes de projectiles et armes de jet. Les végétaux collectés dans les environs étaient travaillés pour confectionner des cordes, des nattes et des filets. Les coquilles étaient consommées, mais aussi utilisées pour la production d'objets de parure ou d'outils que l'on retrouve associés aux morts dans les tombes. 
L'étude archéozoologique des rejets domestiques indique, comme on pouvait s'y attendre, une prédominance des ressources marines dans l'alimentation carnée (poissons, coquillages, tortues et mammifères marins). Les animaux domestiques (moutons, chèvres et bovins) représentent seulement un quart de l'assemblage étudié et la part des animaux chassés est infime [UERPMANN, 2003]. En complément de ces ressources carnées, les occupants du site pouvaient consommer certains végétaux sauvages, comme les jujubes dont on a retrouvé quelques noyaux carbonisés [BIAGI et NISBET, 1992]. Les analyses isotopiques menées sur plusieurs individus viennent cependant relativiser l'apport des végétaux dans l'alimentation, puisqu'elles révèlent un régime alimentaire principalement basé sur la consommation de produits marins, qui suggère une faible mobilité du groupe, ou limitée au littoral [ZAZZO et al., 2014].

L'étude de l'état bucco-dentaire confirme une tendance déjà observée pour la même période chez les pasteurs d'al-Buhais : une alimentation chargée en particules abrasives et qualitativement riche en protéines et faible en glucides [MUNOZ, 2017].

Sur la plupart des sites néolithiques côtiers, l'observation de certains stigmates indique également l'emploi régulier des dents pour des activités extra-alimentaires (travail des fibres et des peaux ou utilisation des dents comme tenaille ou pour le broyage) [MUNOz, 2014]. Enfin, l'analyse des hypoplasies de l'émail dentaire - défauts dus à un stress métabolique pendant le dépôt de la matrice organique - montre que les groupes néolithiques côtiers étaient fréquemment soumis à des épisodes de stress physiologiques. En accord avec ces données, une très forte occurrence de spina bifida a été relevée à Ra's al-Hamra 5 [COPPA et CUCINA, 2007]. Ce défaut d'ossification du sacrum est congénital et peut être imputé soit à un fort taux d'endogamie dans le groupe soit à une carence en acide folique (vitamine B9), abondant dans certains végétaux.

L'ensemble de ces indicateurs suggère la forte dépendance vis-à-vis de ressources dont l'approvisionnement était incertain. La faible diversité des aliments consommés a pu entraîner, chez cette population côtière, des carences alimentaires, voire des épisodes récurrents de disette.

\section{Ancrage territorial et signes de violences}

Dans une région où les ressources sont rares et morcelées, la recherche des lieux les plus propices a visiblement conduit à un ancrage territorial fort. L'implantation durable de communautés dans des zones favorables (sources d'eau, embouchures de wadi, mangroves, lagunes) est soulignée par la durée et l'intensité de l'occupation de certains sites, fréquentés pendant plusieurs siècles et comportant d'importants ensembles funéraires. La pression exercée sur ces ressources et la nécessité d'en assurer le contrôle ont pu conduire à des tensions au sein même des communautés ou entre groupes rivaux.

À al-Buhais, la population a connu des épisodes récurrents de violence, liés à des conflits entre groupes rivaux ou au sein même du groupe : sur 341 crânes observables, $11 \%$ révèlent des traces de traumatismes et certains portent des fractures multiples [KutTERER et UerpmanN, 2012]. Les hommes sont deux fois plus touchés que les femmes et les enfants de moins de 15 ans ne sont pas affectés. Parmi les ossements postcrâniens, 3,3\% présentent des fractures consolidées, principalement localisées sur les os des membres supérieurs. Le site a d'ailleurs révélé une très importante proportion de sépultures multiples $(30 \%)$, dans lesquelles jusqu’à cinq individus ont pu être inhumés simultanément [KIESEWETTER, 2006]. Ces sépultures multiples suggèrent des crises de mortalité fréquentes, dont certaines pourraient être en lien avec des conflits. Dans le même temps, des indices d'une certaine solidarité et de la pratique de soins au sein du groupe sont manifestes, non seulement à travers les traumatismes crâniens cicatrisés, mais aussi par trois cas de trépanations cicatrisées qui attestent de traitements chirurgicaux réussis, suggérant une grande maitrise technique [KIESEWETTER, 2006].

Des épisodes violents ont aussi régulièrement ponctué la vie des communautés de pêcheurs néolithiques 
Munoz O. (2019) "Évolution de l'état sanitaire et stratégies de subsistance des populations protohistoriques en Arabie orientale », In : Froment A. \& Guy H. (Dir.), Archéologie de la santé, anthropologie du soin, Paris, Editions la Découverte/Inrap, pp. 95-106.

occupant les rivages de l'Arabie orientale, comme peuvent en témoigner la pointe de flèche fichée dans une vertèbre à Ra's al-Hamra 5 [Santini, 2002] et la forte incidence de sépultures multiples sur ce site $(20 \%)$ [MUNOZ, 2014] (fig. 2) ainsi que la pointe de flèche retrouvée dans une sépulture multiple de l'émirat d'Umm al-Quwain [MéRY et al., 2016], mais aussi les fractures de " parade » repérées sur les avant-bras des individus du groupe inhumé à Wadi Shab, sur la côte du golfe d'Oman [MunOz, 2014].
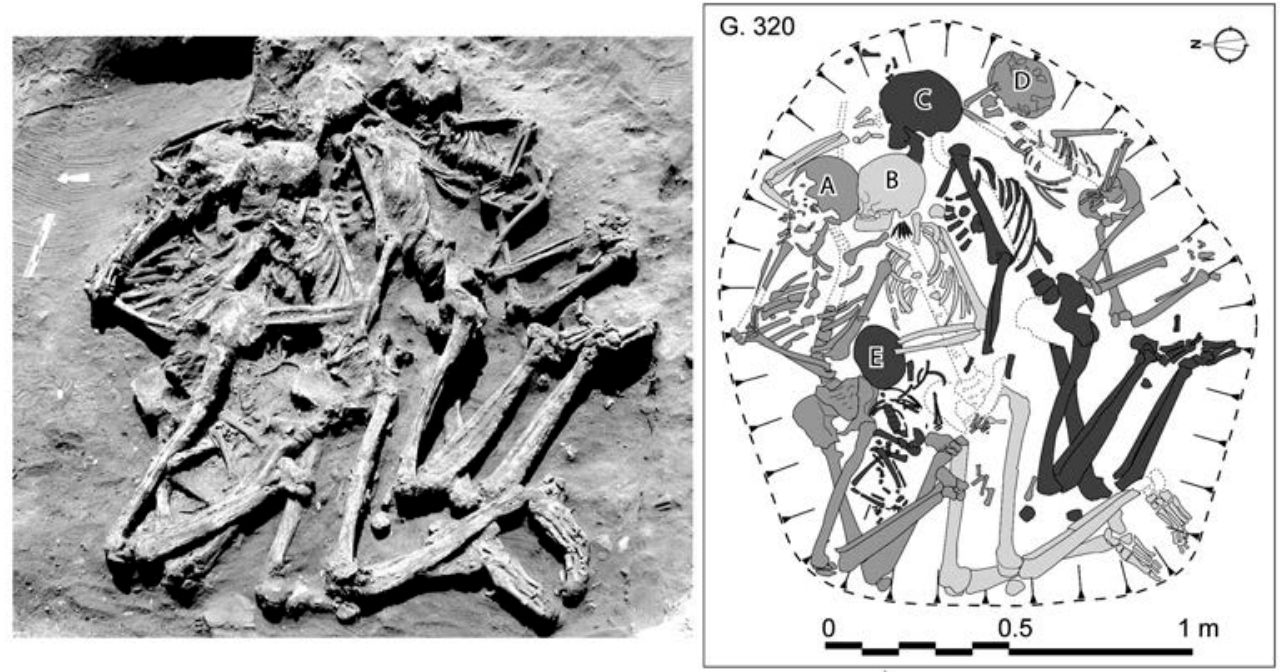

Figure 2 : Sépulture multiple de Ra's al-Hamra 5 (sultanat d'Oman, $\mathrm{IV}^{\mathrm{e}}$ millénaire avant notre ère) où cinq individus ont été inhumés simultanément (Cliché et relevé $\mathrm{O}$. Munoz / Mission archéologique italienne en Oman).

\section{La « grande transformation » de l'âge du Bronze}

Autour de 3000 avant notre ère est amorcée une profonde transformation qui marque la fin du Néolithique et le début de l'âge du Bronze ancien, avec l'apparition de nouvelles techniques telles que la métallurgie et la poterie, le développement de villages à l'architecture permanente et le déploiement de réseaux d'échanges à plusieurs échelles, notamment avec des régions éloignées comme la Mésopotamie, l'Iran ou la vallée de l'Indus [CleUZIOU et TOSI, 2007 ; MÉRY, 2000]. L'âge du Bronze ancien est aussi caractérisé par les mutations importantes qui touchent les pratiques funéraires : les morts ne sont plus inhumés dans des fosses à proximité des habitations comme au Néolithique, mais déposés dans des tombes collectives, construites en pierre, et qui deviennent de plus en plus monumentales au cours du III millénaire avant notre ère [BORTOLINI et MUNOZ, 2015; MÉRY et CHARPENTIER, 2009]. Leur distribution traduit, dès le début du III ${ }^{\mathrm{e}}$ millénaire, une expansion territoriale spectaculaire vers l'arrière-pays. Ces tombes jalonnent en effet le paysage et marquent tout le territoire exploité : côtes rocheuses, plaines côtières, piémonts et vallées.

Dans l'intérieur des terres, le mode de subsistance des populations du début du III ${ }^{e}$ millénaire est encore mal appréhendé, du fait de la rareté des sites d'habitats connus et d'une quasi-absence de données anthropologiques. Il semble que les communautés aient adopté une économie plus diversifiée, intégrant au pastoralisme les premières étapes d'un système agricole basé sur la culture du palmier-dattier. À partir du milieu du III millénaire, de nombreux sites de piémonts attestent de l'installation de villages permanents autour de proto-oasis [Cleuziou, 2002; CHARBOnNier, 2017]. Ainsi par exemple, à Bahla (sultanat d'Oman), une tombe collective datée de la fin du III ${ }^{e}$ millénaire a livré des individus présentant de nombreuses lésions carieuses, des abcès et des pertes de dents ante-mortem. Tous ces indicateurs suggèrent 
que la part des produits agricoles (notamment la datte) était prépondérante dans l'alimentation et significativement plus importante que chez leurs congénères vivant sur la côte [MUNOZ, 2017].

En effet, pour les zones côtières, l'analyse des conditions bucco-dentaires des groupes de Ra's al-Jinz (sultanat d'Oman) fait relativiser fortement le rôle de l'agriculture dans l'économie des populations. Les pathologies clairement imputables à la consommation de produits agricoles n'apparaissent sur la côte que vers le milieu du III ${ }^{e}$ millénaire, et surtout, leur fréquence reste modérée en comparaison des populations de l'intérieur des terres [MUNOZ, 2017]. Les données disponibles pour des sites côtiers du golfe Persique semblent indiquer une diversité de modes de subsistance, alliant la pêche, l'élevage et la consommation de produits agricoles, selon des parts assez variables [LITTLETON et FrOHLICH, 1993 ; GREGORICKA, 2013].

Certaines innovations techniques de l'âge du Bronze ancien semblent avoir eu un impact direct sur la santé des populations quelle que soit la zone géographique concernée. Par exemple, la diminution significative de l'usure dentaire au milieu du III ${ }^{e}$ millénaire suggère des changements dans le mode de préparation des aliments (cuisson bouillie), plus mous et moins abrasifs, que l'on peut attribuer à l'introduction de l'usage de la poterie dans la sphère domestique. Quant aux indicateurs de stress, comme les hypoplasies de l'émail, ils diminuent considérablement dès le début du $\mathrm{III}^{\mathrm{e}}$ millénaire et suggèrent une amélioration globale des conditions de vie, probablement reliée à une meilleure maîtrise de l'approvisionnement et une alimentation plus diversifiée [MUNOZ, 2017]. Les estimations issues des ensembles funéraires de Ra's al-Jinz suggèrent que les communautés connaissent un accroissement démographique, qui s'accélère pendant le III millénaire pour atteindre son apogée vers 2500 avant notre ère [MUNOZ, 2014].

\section{Des perspectives de recherches prometteuses}

En résumé et pour conclure, l'Arabie orientale a suivi, au cours de la préhistoire récente, des trajectoires très différentes de celles des régions voisines du Proche-Orient. Si certains sites occupés pendant plusieurs siècles révèlent un fort ancrage territorial des groupes dès le Néolithique, les fluctuations climatiques et le morcèlement des ressources ont suscité des adaptations basées sur la mobilité et les échanges entre régions complémentaires. Les groupes néolithiques semblent avoir connu un faible accroissement démographique et des crises de mortalité récurrentes, liées à des épisodes de violence, à des épidémies, ou encore à des périodes de disette. Sur les côtes omanaises, les populations se sont spécialisées dans une subsistance presque exclusivement basée sur l'exploitation des ressources marines et côtières.

Avec l'apparition de l'agriculture, cette adaptation côtière perdure, bien qu'il existe des variantes selon les sites, probablement en fonction de la part que prennent les populations locales dans les réseaux d'échanges régionaux. Les denrées agricoles parviennent sur les côtes à l'instar d'autres biens, mais leur rôle dans l'alimentation reste limité en comparaison des sites de l'intérieur, où elle est prépondérante. Il semble que cela ait perduré encore pendant plusieurs millénaires, puisque les voyageurs antiques, du temps d'Alexandre le Grand, qualifiaient d'ichtyophages (se nourrissant de poisson), les peuples qu'ils rencontrèrent en Arabie orientale. L'amélioration des conditions de vie et l'uniformisation culturelle qui marque la deuxième moitié du III ${ }^{\mathrm{e}}$ millénaire est fragile, puisque la fin du Bronze ancien est marquée par de nouveaux bouleversements socio-économiques. Ils sont peut-être en partie liés à la dégradation du climat et à un effondrement des échanges extrarégionaux auxquels les populations locales prenaient part, mais aussi probablement à la déstabilisation des sociétés face à un accroissement des différentiations sociales. Les recherches consacrées à cette transition n'en sont encore qu'à leurs débuts, mais offrent de belles perspectives pour suivre l'évolution sanitaire des populations en Arabie orientale. 


\section{Remerciements}

L'auteure remercie l'ANR « NEOARABIA » (ANR-16-CE03-0007), la mission archéologique française en Oman «Mer d'Arabie (MEAE), la mission italienne en Oman, le LabEx Les passés dans le présent (Investissements d'avenir ref. ANR-11-LABX-0026-01), et le Ministry of Heritage and Culture (MHC) du sultanat d'Oman.

\section{Références bibliographiques}

BORTOLIni E. et MuNOZ O. (2015), «Life and death in prehistoric Oman: Insights from Late Neolithic and Early Bronze Age funerary practices (4th-3rd mill. BC) », The Archaeological Heritage of Oman, Paris, UNESCO / Muscat, sultanat d'Oman, Ministry of Heritage and Culture, p. 61-80.

BEECH M. et al. (2009), "Prehistoric camels in south-eastern Arabia: The discovery of a new site in Abu Dhabi's Western Region, United Arab Emirates ", Proceedings of the Seminar for Arabian Studies, vol. 39, p. 17 30 .

BiAgi P. et NisbET R. (1992), «Environmental history and plant exploitation at the aceramic sites of RH5 and RH6 near the mangrove swamp of Qurum (Muscat - Oman) ", Bulletin de la Société botanique de France, vol. 139 , p. 571-578.

Charbonnier J. (2017), "The genesis of oases in southeast Arabia: Rethinking current theories and models", in LaVIE E. et Marshall A. (éd.), Oases and Globalisation, Springer Geography, p. 53-72.

Cleuziou S. (1998), "The emergence of oases and towns in eastern and southern Arabia », in AfANAS'Ev G. et al. (éd.), The Prehistory of Arabia and Oceania: Proceedings of the XIIIth Congress of the IUPPS, Forlì (Italie), ABACO, p. 159-165.

Cleuziou S. (2002), "Présence et mise en scène des morts à l'usage des vivants dans les communautés préhistoriques : l'exemple de la péninsule d'Oman à l'âge du Bronze ancien ", in MOLINOS M. et ZifFerero A. (éd.), I primi popoli d'Europa, Florence, All'Insegna del Giglio, p. 17-31.

Cleuziou S. (2005), " Pourquoi si tard ? Nous avons pris un autre chemin », in GUILAINE J., Aux marges des grands foyers du Néolithique. Périphéries débitrices ou créatrices?, Paris, Errance, p. 123-148.

Cleuziou S. et TOSI M. (2007), In the Shadow of the Ancestors: The Prehistoric Foundations of the Early Arabian Civilization in Oman, Muscat, sultanat d'Oman, Ministry of Heritage and Culture.

Coppa A. et Cucina A. (2007), "The anthropological analysis ", Journal of Oman Studies, vol. 14, p. 203-342.

GREGORICKA L. A. (2013), "Geographic origins and dietary transitions during the Bronze Age in the Oman Peninsula », American Journal of Physical Anthropology, vol. 152 (3), p. 353-369.

KiesewetTer H. (2006), "Analysis of the human remains from the Neolithic cemetery at al-Buhais 18 (excavations 1996-2000) ", in Uerpmann H.-P., Uerpmann M. et Jasim S. A., The Archaeology of Jebel alBuhais, Sharjah, United Arab Emirates, vol. 1, Tübingen (Allemagne), Kerns Verlag.

KutTerer A. U. (2010), "Remarks on Neolithic burial customs in south-east Arabia », in WeEKs L. (éd.), Death and Burial in Arabia and Beyond, Oxford, Archaeopress (coll. BAR international Series 2107), p. 1-10.

KutTerer A. U. et Uerpmann H.-P. (2012), « Social implication and potential causes of violence at neolithic al-Buhais 18 ", in POTTS D. T. et Hellyer P. (éd.), Fifty years of Emirates Archaeology Proceedings of the Second International Conference on the Archaeology of the United Arab Emirates, Ajman (Émirats arabes unis), Motivate Publishing, p. 55-67.

KUTterer A. U. et Uerpmann H.-P. (2017), « Neolithic nomadism in south-east Arabia - strontium and 
oxygen isotope ratio in human tooth enamel from al-Buhais 18 and Umm al-Quwain 2 in the Emirates of Sharjah and Umm al-Quwain (UAE) ", Arabian Archaeology and Epigraphy, vol. 28, p. 75-89.

LEZINE A.-M. et al. (2010), "Climate change and human occupation in the Southern Arabian lowlands during the last deglaciation and the Holocene ", Global and Planetary Change, vol. 4, p. 412-428.

Littleton J. et Frohlich B. (1993), "Fish-eaters and farmers: Dental pathology in the Arabian Gulf", American Journal of Physical Anthropology, vol. 92 (4), p. 427-447.

MARCUCCI L. G. et al. (2011), "Recent investigations at the prehistoric site RH-5 (Ra's al-Hamra, Muscat, Sultanate of Oman) ", Proceedings of the Seminar for Arabian Studies, 41.

Mery S. (2000), Les Céramiques d'Oman et l'Asie moyenne. Une archéologie des échanges à l'áge du Bronze, Paris, CNRS.

Mery S. et Charpentier V. (2009), « Rites funéraires du Néolithique et de l'âge du Bronze ancien en Arabie orientale ", in Guilaine J. (éd.), Sépultures et sociétés. Du Néolithique à l'Histoire, Paris, Errance, p. 11-40.

MERY S. et al. (2016), «Mort violente en Arabie. La sépulture multiple d'Umm al-Quwain UAQ2 (Émirats arabes unis), VIe millénaire BC», in DUPONT C. et MARCHAND G. (éd.), Archéologie des chasseurs-cueilleurs maritimes De la fonction des habitats à l'organisation de l'espace littoral, Paris, Séances de la Société préhistorique française, vol. 6, p. 323-343.

MunOz O. (2014), «Pratiques funéraires et paramètres biologiques dans la péninsule d'Oman du Néolithique à la fin de l'âge du Bronze ancien (V $V^{e}-I I I{ }^{e}$ millénaire avant notre ère) ", thèse de doctorat, Université de Paris 1 Panthéon-Sorbonne / Università di Roma La Sapienza.

MUNOZ O. (2017), "Transition to agriculture in south-eastern Arabia: Insights from oral conditions ", American Journal of Physical Anthropology, vol. 164 (4), p. 702-719.

SALVATORI S. (2007), "The Prehistoric Graveyard of Ra's al-Hamra 5, Muscat, Sultanate of Oman », The Journal of Oman Studies, vol. 14, p. 5-202.

SANTINI G. (2002), "Burial complex 43 at the prehistoric graveyard of Ra's al-Hamra in northern Oman ", in Cleuziou S., Tosi M. et Zarins J. (éd.), Arabia Antiqua, Essays on the Late Prehistory of the Arabian Peninsula, Rome, IsIAO, p. 147-167.

TengBerg M. (2012), "Beginnings and early history of date palm garden cultivation in the Middle East ", Journal of Arid Environments, vol. 86, p. 139-147.

Uerpmann M. (2003), "The Dark Millennium - Remarks on the Final Stone Age in the Emirates and Oman ", in PotTs D. T., Al Nabooda H. et Hellyer P., Archaeology of the United Arab Emirates, Londres, Trident Press, p. 74-81.

Uerpmann H.-P. et Uerpmann M. (2002), "The Appearance of the Domestic Camel in South-east Arabia », Journal of Oman Studies, vol. 12, p. 235-260.

Uerpmann H.-P., Uerpmann M. et Jasim S. A. (2006), The Archaeology of Jebel al-Buhais, Sharjah, United Arab Emirates. I. Funeral Monuments and Human Remains from Jebel al-Buhais, Tübingen (Allemagne), Kerns Verlag.

Uerpmann H.-P., Uerpmann M. et Jasim S. A. (2008), The Archaeology of Jebel al-Buhais, Sharjah, United Arab Emirates. II. The Natural Environment of Jebel al-Buhais: Past and Present, Tübingen (Allemagne), Kerns Verlag.

ZAZzo A., Munoz O. et SALIĖGE J. F. (2014), «Diet and mobility in a late Neolithic population of coastal Oman inferred from radiocarbon dating and stable isotope analysis ", American Journal of Physical Anthropology, vol. 153 (3), p. 353-364. 